\title{
The excluded minor structure theorem with planarly embedded wall
}

\author{
Bojan Mohar* \\ Department of Mathematics, Simon Fraser University, Burnaby, B.C. V5A 1S6, Canada
}

Received 25 September 2009, accepted 29 May 2012, published online 11 July 2012

\begin{abstract}
A graph is "nearly embedded" in a surface if it consists of graph $G_{0}$ that is embedded in the surface, together with a bounded number of vortices having no large transactions. It is shown that every large wall (or grid minor) in a nearly embedded graph, many rows of which intersect the embedded subgraph $G_{0}$ of the near-embedding, contains a large subwall that is planarly embedded within $G_{0}$. This result provides some hidden details needed for a strong version of the Robertson and Seymour's excluded minor theorem as presented in [1].
\end{abstract}

Keywords: Graph, graph minor, surface, near-embedding, grid minor, excluded minor.

Math. Subj. Class.: 05C10, 05C82

\section{Introduction}

A graph is a minor of another graph if the first can be obtained from a subgraph of the second by contracting edges. One of the highlights of the graph minors theory developed by Robertson and Seymour is the Excluded Minor Theorem (EMT) that describes a rough structure of graphs that do not contain a fixed graph $H$ as a minor. Two versions of EMT appear in [7, 8]; see also [3] and [4].

In [1] and [2] the authors used a strong version of EMT in which it is concluded that every graph without a fixed minor and whose tree-width is large has a tree-like structure, whose pieces are subgraphs that are almost embedded in some surface, and in which one of the pieces contains a large grid minor that is (essentially) embedded in a disk on the surface. Although not explicitly mentioned, this version of EMT follows from the published results of Robertson and Seymour [8] by applying standard techniques of routings on surfaces.

\footnotetext{
* Supported in part by ARRS, Research Program P1-0297, by an NSERC Discovery Grant and by the Canada Research Chair program. On leave from Department of Mathematics, University of Ljubljana, Ljubljana, Slovenia.

E-mail address: mohar@sfu.ca (Bojan Mohar)
} 
Experts in this area are familiar with these techniques (that are also present in Robertson and Seymour's work [6]). However, they may be harder to digest for newcomers in the area, and thus deserve to be presented in the written form. The purpose of this note is to provide a proof of an extended version of EMT as stated in [1, Theorem 4.2].

It may be worth mentioning that the proof in [1] does not really need the extended version of the EMT, but the proof in [2] does. Thus, this note may also be viewed as a support for the main proof in [2].

We assume that the reader is familiar with the basic notions of graph theory and in particular with the basic notions related to graph minors; we refer to [3] for all terms and results not explained here.

\section{Walls in near-embeddings}

In this section, we present our main lemma, which shows that for every large wall (to be defined in the sequel) in a "nearly embedded" graph, a large subwall must be contained in the embedded subgraph of the near-embedding. Let us first introduce the notion of the wall and some of its elementary properties.

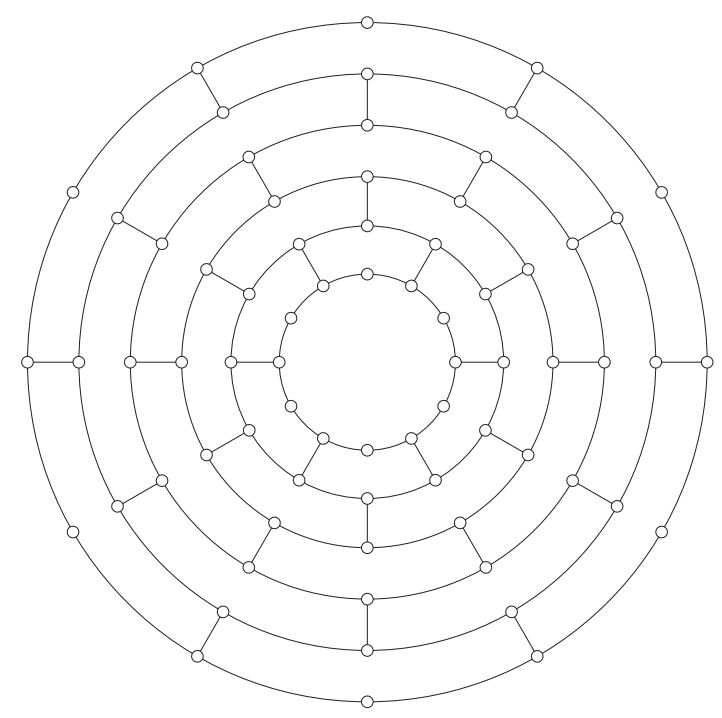

Figure 1: The cylindrical 6-wall $Q_{6}$

For an integer $r \geq 3$, we define a cylindrical $r$-wall as a graph that is isomorphic to a subdivision of the graph $Q_{r}$ defined as follows. We start with vertex set $V=\{(i, j) \mid 1 \leq$ $i \leq r, 1 \leq j \leq 2 r\}$, and make two vertices $(i, j)$ and $\left(i^{\prime}, j^{\prime}\right)$ adjacent if and only if one of the following possibilities holds:

(1) $i^{\prime}=i$ and $j^{\prime} \in\{j-1, j+1\}$, where the values $j-1$ and $j+1$ are considered modulo $2 r$.

(2) $j^{\prime}=j$ and $i^{\prime}=i+(-1)^{i+j}$.

Less formally, $Q_{r}$ consists of $r$ disjoint cycles $C_{1}, \ldots, C_{r}$ of length $2 r$ (where $V\left(C_{i}\right)=$ 
$\{(i, j) \mid 1 \leq j \leq 2 r\}$ ), called the meridian cycles of $Q_{r}$. Any two consecutive cycles $C_{i}$ and $C_{i+1}$ are joined by $r$ edges so that the edges joining $C_{i}$ and $C_{i-1}$ interlace on $C_{i}$ with those joining $C_{i}$ and $C_{i+1}$ for $1<i<r$. Figure 1 shows the cylindrical 6-wall $Q_{6}$.

By deleting the edges joining vertices $(i, 1)$ and $(i, 2 r)$ for $i=1, \ldots, r$, we obtain a subgraph of $Q_{r}$. Any graph isomorphic to a subdivision of this graph is called an $r$-wall.

To relate walls and cylindrical walls to $(r \times r)$-grid minors, we state the following easy correspondence:

(a) Every $(4 r+2)$-wall contains a cylindrical $r$-wall as a subgraph.

(b) Every cylindrical $r$-wall contains an $(r \times r)$-grid as a minor.

(c) Every $(r \times r)$-grid minor contains an $\left\lfloor\frac{r-1}{2}\right\rfloor$-wall as a subgraph.

Lemma 2.1. Suppose that $1 \leq i<j \leq r$ and let $t=j-i-1$. Let $S_{i} \subset C_{i}$ and $S_{j} \subseteq C_{j}$ be paths of length at least $2 t-1$ in the meridian cycles $C_{i}, C_{j}$ of $Q_{r}$. Then $Q_{r}$ contains $t$ disjoint paths linking $C_{i}$ and $C_{j}$. Moreover, for each of these paths and for every cycle $C_{k}$, $i<k<j$, the intersection of the path with $C_{k}$ is a connected segment of $C_{k}$.

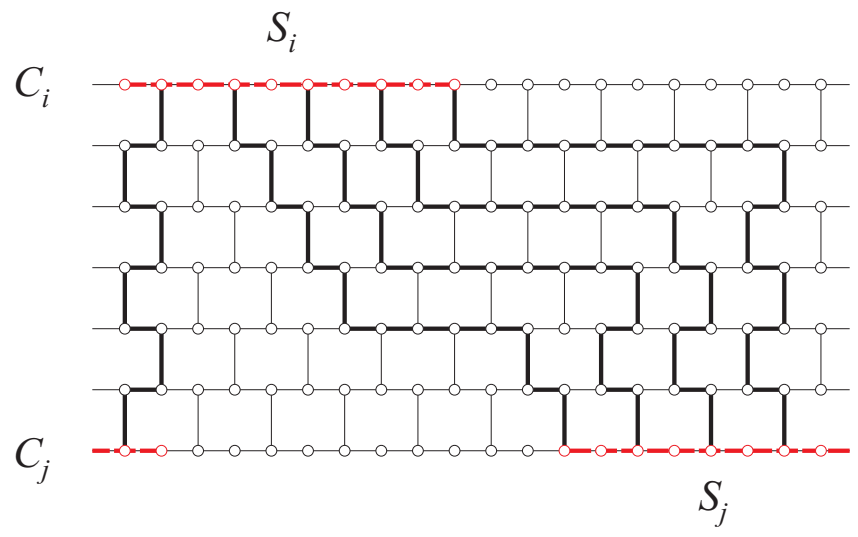

Figure 2: Paths linking $S_{i}$ and $S_{j}$

Proof. The lemma is easy to prove and the idea is illustrated in Figure 2, in which the edges on the left are assumed to be identified with the corresponding edges on the right. The paths are shown by thick lines and the segments $S_{i}$ and $S_{j}$ are shown by thick broken lines.

A surface is a compact connected 2-manifold (with or without boundary). The components of the boundary are called the cuffs. If a surface $S$ has Euler characteristic $c$, then the non-negative number $g=2-c$ is called the Euler genus of $S$. Note that a surface of Euler genus $g$ contains at most $g$ cuffs.

Disjoint cycles $C, C^{\prime}$ in a graph embedded in a surface $S$ are homotopic if there is a cylinder in $S$ whose boundary components are the cycles $C$ and $C^{\prime}$. The cylinder bounded by homotopic cycles $C, C^{\prime}$ is denoted by $\operatorname{int}\left(C, C^{\prime}\right)$. Disjoint paths $P, Q$ whose initial vertices lie in the same cuff $C$ and whose terminal vertices lie in the same cuff $C^{\prime}$ in $S$ 
(possibly $C^{\prime}=C$ ) are homotopic if $P$ and $Q$ together with a segment in $C$ and a segment in $C^{\prime}$ form a contractible closed curve $A$ in $S$. The disk bounded by $A$ will be denoted by $\operatorname{int}(P, Q)$. The following basic fact about homotopic curves on a surface will be used throughout (cf., e.g., [5, Propositions 4.2.6 and 4.2.7]).

Lemma 2.2. Let $S$ be a surface of Euler genus $g$. Then every collection of more than $3 g$ disjoint non-contractible cycles contains two cycles that are homotopic. Similarly, every collection of more than $3 g$ disjoint paths, whose ends are on the same (pair of) cuffs in $S$, contains two paths that are homotopic.

Let $G$ be a graph and let $W=\left\{w_{0}, \ldots, w_{n}\right\}, n=|W|-1$, be a linearly ordered subset of its vertices such that $w_{i}$ precedes $w_{j}$ in the linear order if and only if $i<j$. The pair $(G, W)$ is called a vortex of length $n, W$ is the society of the vortex and all vertices in $W$ are called society vertices. When an explicit reference to the society is not needed, we will as well say that $G$ is a vortex. A collection of disjoint paths $R_{1}, \ldots, R_{k}$ in $G$ is called a transaction of order $k$ in the vortex $(G, W)$ if there exist $i, j(0 \leq i \leq j \leq n)$ such that all paths have their initial vertices in $\left\{w_{i}, w_{i+1}, \ldots, w_{j}\right\}$ and their endvertices in $W \backslash\left\{w_{i}, w_{i+1}, \ldots, w_{j}\right\}$.

Let $G$ be a graph that can be expressed as $G=G_{0} \cup G_{1} \cup \cdots \cup G_{v}$, where $G_{0}$ is embedded in a surface $S$ of Euler genus $g$ with $v$ cuffs $\Omega_{1}, \ldots, \Omega_{v}$, and $G_{i}(i=1, \ldots, v)$ are pairwise disjoint vortices, whose society is equal to their intersection with $G_{0}$ and is contained in the cuff $\Omega_{i}$, with the order of the society being inherited from the circular order around the cuff. Then we say that $G$ is near-embedded in the surface $S$ with vortices $G_{1}, \ldots, G_{v}$. A subgraph $H$ of a graph $G$ that is near-embedded in $S$ is said to be planarly embedded in $S$ if $H$ is contained in the embedded subgraph $G_{0}$, and there exists a cycle $C \subseteq G_{0}$ that is contractible in $S$ and $H$ is contained in the disk on $S$ that is bounded by $C$. Our main result is the following.

Theorem 2.3. For every non-negative integers $g, v, a$ there exists a positive integer $s=$ $s(g, v, a)$ such that the following holds. Suppose that a graph $G$ is near-embedded in the surface $S$ with vortices $G_{1}, \ldots, G_{v}$, such that the maximum order of transactions of the vortices is at most $a$. Let $Q$ be a cylindrical $r$-wall contained in $G$, such that at least $r_{0} \geq 3 s$ of its meridian cycles have at least one edge contained in $G_{0}$. Then $Q \cap G_{0}$ contains a cylindrical $r^{\prime}$-wall that is planarly embedded in $S$ and has $r^{\prime} \geq r_{0} / s$.

Proof. Let $C_{p_{1}}, C_{p_{2}}, \ldots, C_{p_{r_{0}}}\left(p_{1}<p_{2}<\cdots<p_{r_{0}}\right)$ be meridian cycles of $Q$ having an edge in $G_{0}$. For $i=1, \ldots, r_{0}$, let $L_{i}$ be a maximal segment of $C_{p_{i}}$ containing an edge in $E\left(C_{p_{i}}\right) \cap E\left(G_{0}\right)$ and such that none of its vertices except possibly the first and the last vertex are on a cuff. It may be that $L_{i}=C_{p_{i}}$ if $C_{p_{i}}$ contains at most one vertex on a cuff; if not, then $L_{i}$ starts on some cuff and ends on (another or the same) cuff. (We think of the meridian cycles to have the orientation as given by the meridians in the wall.) At least $r_{0} /\left(v^{2}+1\right)$ of the segments $L_{i}$ either start and end up on the same cuffs $\Omega_{x}$ and $\Omega_{y}$ (possibly $x=y$ ), or are all cycles. In each case, we consider their homotopies. By Lemma 2.2 , these segments contain a subset of $q \geq r_{0} /\left((3 g+1)\left(v^{2}+1\right)\right)$ homotopic segments (or cycles). Since we will only be interested in these homotopic segments or cycles, we will assume henceforth that $L_{1}, \ldots, L_{q}$ are homotopic.

Let us first look at the case when $L_{1}, \ldots, L_{q}$ are cycles. Since $s=s(g, v, a)$ can be chosen to be arbitrarily large (as long as it only depends on the parameters), we may assume 


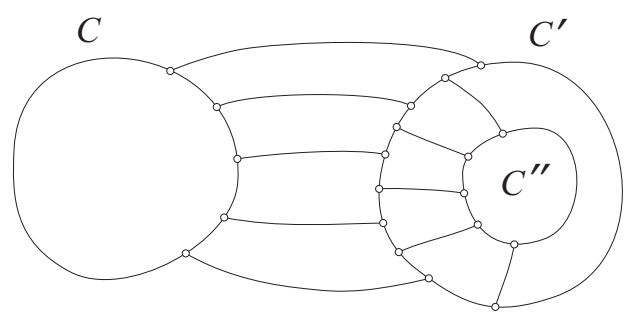

Figure 3: Many contractible cycles

that $q$ is as large as needed in the sequel. If the cycles $L_{i}$ are pairwise homotopic and noncontractible, then it is easy to see that two of them bound a cylinder in $S$ containing many of these cycles. This cylinder also contains the paths connecting these cycles; thus it contains a large planarly embedded wall and hence also a large planarly embedded cylindrical wall. So, we may assume that the cycles $L_{1}, \ldots, L_{q}$ are contractible. By Lemma 2.1, $Q$ contains $t$ paths linking any two of these cycles that are $t$ apart in $Q$, say $C=L_{i}$ and $C^{\prime}=L_{i+t+1}$. (Here we take $t$ large enough that the subsequent arguments will work.) Again, many of these paths either reach $C^{\prime}$ without intersecting any of the cuffs, or many reach the same cuff $\Omega$. A large subset of them is homotopic. In the former case, the paths linking $C^{\prime}$ with $C^{\prime \prime}=L_{i+2 t+2}$ can be chosen so that their initial vertices interlace on $C^{\prime}$ with the endvertices of the homotopic paths coming from $C$. This implies that $C$ or $C^{\prime \prime}$ lies in the disk bounded by $C^{\prime}$ (cf. Figure 3). By repeating the argument, we obtain a sequence of nested cycles and interlaced linkages between them. This clearly gives a large subwall, which contains a large cylindrical subwall that is planarly embedded. In the latter case, when the paths from $C$ to $C^{\prime}$ go through the same cuff $\Omega_{j}$, we get a contradiction since the vortex on $\Omega_{j}$ does not admit a transaction of large order, and thus too many homotopic paths cannot reach $C^{\prime \prime}$.

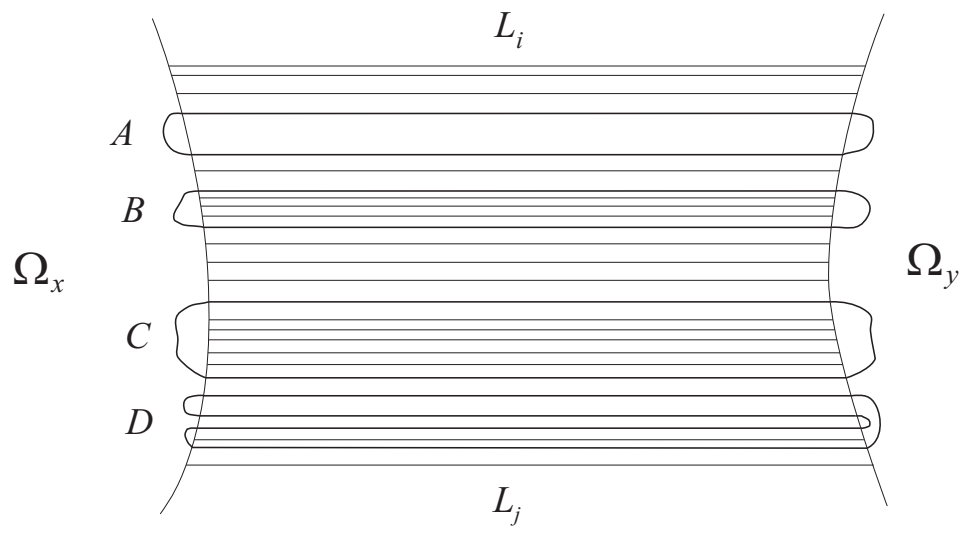

Figure 4: Many homotopic segments joining two cuffs

We get a similar contradiction as in the last case above, when too many homotopic segments $L_{i}$ start and end up on the same cuffs $\Omega_{x}$ and $\Omega_{y}$. We shall give details for the 
case when $x \neq y$, but the same approach works also if $x=y$. (In the case when $x=y$ and the homotopic segments $L_{i}$ are contractible, the proof is similar to the part of the proof given above.)

Let us consider the "extreme" segments $L_{i}, L_{j}$, whose disk $\operatorname{int}\left(L_{i}, L_{j}\right)$ contains many homotopic segments (cf. Figure 4). Let us enumerate these segments as $L_{1}^{\prime}=L_{i}, L_{2}^{\prime}, \ldots$, $L_{m}^{\prime}=L_{j}$ in the order as they appear inside $\operatorname{int}\left(L_{i}, L_{j}\right)$. Let $C_{t}^{\prime}$ (for $1<t<m$ ) be the meridian cycle containing the segment $L_{t}^{\prime}$. Since vortices admit no transactions of order more than $a$, at most $4 a$ of the cycles $C_{t}^{\prime}(1<t<m)$ can leave $\operatorname{int}\left(L_{i}, L_{j}\right)$. By adjusting $m$, we may thus assume that none of them does. In particular, each $L_{t}^{\prime}$ has another homotopic segment in $\operatorname{int}\left(L_{i}, L_{j}\right)$. Since there are no transactions of order more than $a$, there is a large subset of the cycles $C_{t}^{\prime}$ that follow each other in $\operatorname{int}\left(L_{i}, L_{j}\right)$ as shown by the thick cycles in Figure 4. Consider four of these meridian cycles $A, B, C, D$ that are pairwise far apart in the wall $Q$ and appear in the order $A, B, C, D$ within $\operatorname{int}\left(L_{i}, L_{j}\right)$. Then $A$ and $C$ are linked in $Q$ by a large collection of disjoint paths by Lemma 2.1. At most $8 a$ of these paths can escape intersecting two fixed segments $L_{u}^{\prime}$ and $L_{v}^{\prime}$ of $B$ or two such segments of $D$ by passing through a vortex. All other paths linking $A$ and $C$ intersect either two segments of $B$ or two segments of $D$. However, this is a contradiction since the paths linking $A$ and $C$ can be chosen in $Q$ so that each of them intersects each meridian cycle in a connected segment (Lemma 2.1). This completes the proof.

\section{The excluded minor structure}

In this section, we define some of the structures found in Robertson-Seymour's Excluded Minor Theorem [7] which describes the structure of graphs that do no contain a given graph as a minor. Robertson and Seymour proved a strengthened version of that theorem that gives a more elaborate description of the structure in [8]. Our terminology follows that introduced in [1].

Let $G_{0}$ be a graph. Suppose that $\left(G_{1}^{\prime}, G_{2}^{\prime}\right)$ is a separation of $G_{0}$ of order $t \leq 3$, i.e., $G_{0}=G_{1}^{\prime} \cup G_{2}^{\prime}$, where $G_{1}^{\prime} \cap G_{2}^{\prime}=\left\{v_{1}, \ldots, v_{t}\right\} \subset V\left(G_{0}\right), 1 \leq t \leq 3, V\left(G_{2}^{\prime}\right) \backslash V\left(G_{1}^{\prime}\right) \neq \emptyset$. Let us replace $G_{0}$ by the graph $G^{\prime}$, which is obtained from $G_{1}^{\prime}$ by adding all edges $v_{i} v_{j}$ $(1 \leq i<j \leq t)$ if they are not already contained in $G_{1}^{\prime}$. We say that $G^{\prime}$ has been obtained from $G_{0}$ by an elementary reduction. If $t=3$, then the 3 -cycle $T=v_{1} v_{2} v_{3}$ in $G^{\prime}$ is called the reduction triangle. Every graph $G^{\prime \prime}$ that can be obtained from $G_{0}$ by a sequence of elementary reductions is a reduction of $G_{0}$.

We say that a graph $G_{0}$ can be embedded in a surface $\Sigma$ up to 3-separations if there is a reduction $G^{\prime \prime}$ of $G_{0}$ such that $G^{\prime \prime}$ has an embedding in $\Sigma$ in which every reduction triangle bounds a face of length 3 in $\Sigma$.

Let $H$ be an $r$-wall in the graph $G_{0}$ and let $G^{\prime \prime}$ be a reduction of $G_{0}$. We say that the reduction $G^{\prime \prime}$ preserves $H$ if for every elementary reduction used in obtaining $G^{\prime \prime}$ from $G_{0}$, at most one vertex of degree 3 in $H$ is deleted. (With the above notation, $G_{2}^{\prime} \backslash G_{1}^{\prime}$ contains at most one vertex of degree 3 in $H$.)

Lemma 3.1. Suppose that $G^{\prime \prime}$ is a reduction of the graph $G_{0}$ and that $G^{\prime \prime}$ preserves an $r$-wall $H$ in $G_{0}$. Then $G^{\prime \prime}$ contains an $\lfloor(r+1) / 3\rfloor$-wall, all of whose edges are contained in the union of $H$ and all edges added to $G^{\prime \prime}$ when performing elementary reductions.

Proof. Let $H^{\prime}$ be the subgraph of the $r$-wall $H$ obtained by taking every third row and every third "column". See Figure 5 in which $H^{\prime}$ is drawn with thick edges. It is easy to 


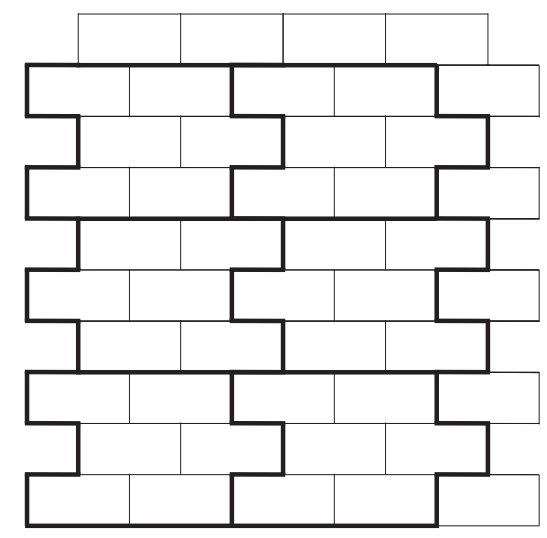

Figure 5: Smaller wall contained in a bigger wall

see that for every elementary reduction we can keep a subgraph homeomorphic to $H^{\prime}$ by replacing the edges of $H^{\prime}$ which may have been deleted by adding some of the edges $v_{i} v_{j}$ involved in the reduction. The only problem would occur when we lose a vertex of degree 3 and when all vertices $v_{1}, v_{2}, v_{3}$ involved in the elementary reduction would be of degree 3 in $H^{\prime}$. However, this is not possible since $G^{\prime \prime}$ preserves $H$.

Suppose that for $i=0, \ldots, n$, there exist vertex sets, called parts, $X_{i} \subseteq V(G)$, with the following properties:

(V1) $X_{i} \cap W=\left\{w_{i}, w_{i+1}\right\}$ for $i=0, \ldots, n$, where $w_{n+1}=w_{n}$,

(V2) $\bigcup_{0 \leq i \leq n} X_{i}=V(G)$,

(V3) every edge of $G$ has both endvertices in some $X_{i}$, and

(V4) if $i \leq j \leq k$, then $X_{i} \cap X_{k} \subseteq X_{j}$.

Then the family $\left(X_{i} ; i=0, \ldots, n\right)$ is called a vortex decomposition of the vortex $(G, W)$. For $i=1, \ldots, n$, denote by $Z_{i}=\left(X_{i-1} \cap X_{i}\right) \backslash W$. The adhesion of the vortex decomposition is the maximum of $\left|Z_{i}\right|$, for $i=1, \ldots, n$. The vortex decomposition is linked if for $i=1, \ldots, n-1$, the subgraph of $G$ induced on the vertex set $X_{i} \backslash W$ contains a collection of disjoint paths linking $Z_{i}$ with $Z_{i+1}$. Clearly, in that case $\left|Z_{i}\right|=\left|Z_{i+1}\right|$, and the paths corresponding to $Z_{i} \cap Z_{i+1}$ are trivial. Note that (V1) and (V3) imply that there are no edges between nonconsecutive society vertices of the vortex. Let us remark that every vortex $(G, W)$, in which $w_{i}, w_{j}$ are non-adjacent for $|i-j| \geq 2$, admits a linked vortex decomposition; just take $X_{i}=(V(G) \backslash W) \cup\left\{w_{i}, w_{i+1}\right\}$.

The (linked) adhesion of the vortex is the minimum adhesion taken over all (linked) decompositions of the vortex. Let us observe that in a linked decomposition of adhesion $q$, there are $q$ disjoint paths linking $Z_{1}$ with $Z_{n}$ in $G-W$. For us it is important to note that a vortex with adhesion less than $k$ does not admit a transaction of order more than $k$.

Let $G$ be a graph, $H$ an $r$-wall in $G, \Sigma$ a surface, and $\alpha \geq 0$ an integer. We say that $G$ can be $\alpha$-nearly embedded in $\Sigma$ if there is a set of at most $\alpha$ cuffs $C_{1}, \ldots, C_{b}(b \leq \alpha)$ in $\Sigma$, and there is a set $A$ of at most $\alpha$ vertices of $G$ such that $G-A$ can be written 
as $G_{0} \cup G_{1} \cup \cdots \cup G_{b}$ where $G_{0}, G_{1}, \ldots, G_{b}$ are edge-disjoint subgraphs of $G$ and the following conditions hold:

(N1) $G_{0}$ can be embedded in $\Sigma$ up to 3 -separations with $G^{\prime \prime}$ being the corresponding reduction of $G_{0}$.

(N2) If $1 \leq i<j \leq b$, then $V\left(G_{i}\right) \cap V\left(G_{j}\right)=\emptyset$.

(N3) $W_{i}=V\left(G_{0}\right) \cap V\left(G_{i}\right)=V\left(G^{\prime \prime}\right) \cap C_{i}$ for every $i=1, \ldots, b$.

(N4) For every $i=1, \ldots, b$, the pair $\left(G_{i}, W_{i}\right)$ is a vortex of adhesion less than $\alpha$, where the ordering of $W_{i}$ is consistent with the (cyclic) order of these vertices on $C_{i}$.

The vertices in $A$ are called the apex vertices of the $\alpha$-near embedding. The subgraph $G_{0}$ of $G$ is said to be the embedded subgraph with respect to the $\alpha$-near embedding and the decomposition $G_{0}, G_{1}, \ldots, G_{b}$. The pairs $\left(G_{i}, W_{i}\right), i=1, \ldots, b$, are the vortices of the $\alpha$ near embedding. The vortex $\left(G_{i}, W_{i}\right)$ is said to be attached to the cuff $C_{i}$ of $\Sigma$ containing $W_{i}$.

If $G$ is $\alpha$-near-embedded in $S$, let $G_{0}, G_{1}, \ldots, G_{b}$ be as above and let $G^{\prime \prime}$ be the reduction of $G_{0}$ that is embedded in $S$. If $H$ is an $r$-wall in $G$, we say that $H$ is captured in the embedded subgraph $G_{0}$ of the $\alpha$-near-embedding if $H$ is preserved in the reduction $G^{\prime \prime}$ and for every separation $G=K \cup L$ of order less than $r$, where $G_{0} \subseteq K$, at least $\frac{2}{3}$ of the degree-3 vertices of $H$ lie in $K$.

We shall use the following theorem which is a simplified version of one of the cornerstones of Robertson and Seymour's theory of graph minors, the Excluded Minor Theorem, as stated in [8]. For a detailed explanation of how the version in this paper can be derived from the version in [8], see the appendix of [1].

Theorem 3.2 (Excluded Minor Theorem). For every graph $R$, there is a constant $\alpha$ such that for every positive integer $w$, there exists a positive integer $r=r(R, \alpha, w)$, which tends to infinity with $w$ for any fixed $R$ and $\alpha$, such that every graph $G$ that does not contain an $R$-minor either has tree-width at most $w$ or contains an $r$-wall $H$ such that $G$ has an $\alpha$-near embedding in some surface $\Sigma$ in which $R$ cannot be embedded, and $H$ is captured in the embedded subgraph of the near-embedding.

We can add the following assumptions about the $r$-wall in Theorem 3.2.

Theorem 3.3. It may be assumed that the $r$-wall $H$ in Theorem 3.2 has the following properties:

(a) $H$ is contained in the reduction $G^{\prime \prime}$ of the embedded subgraph $G_{0}$.

(b) $H$ is planarly embedded in $\Sigma$, i.e., every cycle in $H$ is contractible in $\Sigma$ and the outer cycle of $H$ bounds a disk in $\Sigma$ that contains $H$.

(c) We may prespecify any constant $\rho$ and ask that the face-width of $G^{\prime \prime}$ be at least $\rho$.

(d) $G^{\prime \prime}$ is 3 -connected.

Proof. The starting point is Theorem 3.2. By making additional elementary reductions if necessary, we can achieve (d). The property (c) is attained as follows. If the face-width is too small, then there is a set of less than $\rho$ vertices whose removal reduces the genus of the embedding of $G^{\prime \prime}$. We can add these vertices in the apex set and repeat the procedure as 
long as the face-width is still smaller than $\rho$. The only subtlety here is that the constant $\alpha$ in Theorem 3.2 now depends not only on $R$ but also on $\rho$. See also [4].

After removing the apex set $A$, we are left with an $(r-\alpha)$-wall in $G-A$. By applying Lemma 3.1, we may assume that $H$ is contained in the reduced graph $G^{\prime \prime} \cup G_{1} \cup \cdots \cup G_{b}$. The wall $H$ contains a large cylindrical wall $Q$. Since the vortices have bounded adhesion, they do not have large transactions. Since the wall is captured in $G^{\prime \prime}$, edges of many meridians of $Q$ lie in $G^{\prime \prime}$. Therefore, we can apply Theorem 2.3 for the near embedding of the reduced graph together with the vortices. This shows that a large cylindrical subwall of $Q$ is planarly embedded in the surface. The size $r^{\prime}$ of this smaller wall still satisfies the condition that $r^{\prime}=r^{\prime}(R, \alpha, w) \rightarrow \infty$ as $w$ increases.

It is worth mentioning that there are other ways to show that a graph with large enough tree-width that does not contain a fixed graph $R$ as a minor contains a subgraph that is $\alpha$-near-embedded in some surface $\Sigma$ in which $R$ cannot be embedded, and moreover, there is an $r$-wall planarly embedded in $\Sigma$ (after reductions taking care of at most 3-separations). Let us describe two of them:

(A) Large face-width argument: One can use property (c) in Theorem 3.3 that the facewidth $\rho$ can be made as large as we want if $\alpha=\alpha(R, w, \rho)$ is large enough. Once we have that, it follows from [6] that there is a planarly embedded $r$-wall, where $r=r(R, \rho) \rightarrow \infty$ as $\rho \rightarrow \infty$. While this easy argument is sufficient for most applications, it appears to be slightly weaker than Theorem 3.3 since the quantifiers change. The difference is that the number of apex vertices is no longer bounded as a function of $\alpha=\alpha(R)$ but rather as a function depending on $R$ and $r$, where the upper bound has linear dependence on $r$, i.e. it is of the form $\beta(R) r$. However, other parameters of the near-embedding keep being only dependent on $R$.

(B) Irrelevant vertex: The third way of establishing the same result is to go through the proof of Robertson and Seymour that there is an irrelevant vertex, i.e. a vertex $v$ such that $G$ has an $R$-minor if and only if $G-v$ has. (Compared to the later, more abstract parts of the graph minors series of papers, this part is very clean and well understood; it could (and should) be explained in a(ny) serious graduate course on graph minors.) In that proof, one starts with an arbitrary wall $W$ that is large enough. A large wall exists since the tree-width is large. Then one compares the $W$-bridges attached to $W$. They may give rise to $\leq 3$-separations, to jumps (paths in bridges whose addition to $W$ yields a nonplanar graph), crosses (pairs of disjoint paths attached to the same planar face of $W$ whose addition to $W$ yields a nonplanar graph). If there are many disjoint jumps or crosses on distinct faces of $W$, one can find an $R$-minor. If there are just a few, there is a large planar wall. If there are many of them on the same face, we get a structure of a vortex with bounded transactions (or else an $R$-minor can be discovered). The proof then discusses ways for many jumps and crosses but no large subset of them being disjoint. One way is to have a small set of vertices whose removal destroys most of these jumps and crosses. This gives rise to the apex vertices. The final conclusion is that the jumps and crosses can affect only a bounded part of the wall, so after the removal of the apex vertices and after elementary reductions which eliminate $\leq 3$-separations, there is a large subwall $W_{0}$ such that no jumps or crosses are involved in it. The "middle" vertex in $W_{0}$ is then shown to be irrelevant. 
For our reference, only this planar wall is needed. By being planar, we mean that the rest of the graph is attached only to the outer face of this wall. Then we define the tangle corresponding to this wall and the proof of the EMT preserves this tangle while making the modifications yielding to an $\alpha$-near-embedding.

\section{References}

[1] T. Böhme, K. Kawarabayashi, J. Maharry and B. Mohar, Linear connectivity forces large complete bipartite minors, J. Combin. Theory, Ser. B 99 (2009), 557-582.

[2] T. Böhme, K. Kawarabayashi, J. Maharry and B. Mohar, $K_{3, k}$-minors in large 7 -connected graphs, submitted to J. Combin. Theory, Ser. B, May 2008.

[3] R. Diestel, Graph Theory, 3rd Edition, Springer, 2005.

[4] K. Kawarabayashi and B. Mohar, Some recent progress and applications in graph minor theory, Graphs Combin. 23 (2007), 1-46.

[5] B. Mohar and C. Thomassen, Graphs on Surfaces, Johns Hopkins University Press, Baltimore, MD, 2001.

[6] N. Robertson and P. D. Seymour, Graph minors. VII. Disjoint paths on a surface, J. Combin. Theory Ser. B 45 (1988), 212-254.

[7] N. Robertson and P. D. Seymour, Graph minors. XVI. Excuding a non-planar graph, J. Combin. Theory Ser. B 89 (2003), 43-76.

[8] N. Robertson and P. D. Seymour, Graph minors. XVII. Taming a vortex, J. Combin. Theory Ser. B 77 (1999), 162-210. 